\title{
Risk factors for quinolone-resistant Escherichia coli in feces from preweaned dairy calves and postpartum dairy cows
}

\author{
Anna Duse,${ }^{*} \dagger^{1}$ Karin Persson Waller, ${ }^{*} \dagger$ Ulf Emanuelson, $\dagger$ Helle Ericsson Unnerstad, ${ }^{*}$ Ylva Persson, $\ddagger$ \\ and Björn Bengtsson* \\ *Department of Animal Health and Antimicrobial Strategies, National Veterinary Institute, SE-751 89 Uppsala, Sweden \\ †Department of Clinical Sciences, Swedish University of Agricultural Sciences, SE-750 07 Uppsala, Sweden \\ ¥Växa Sverige, SE-751 05 Uppsala, Sweden
}

\section{ABSTRACT}

Quinolone resistance may emerge in gut bacteria (e.g., in Escherichia coli) of animals. Such bacteria could cause infections in the animal itself or be transmitted to humans via the food chain. Quinolone resistance is also observed in fecal $E$. coli of healthy dairy cattle, but the prevalence varies between farms, not solely as a result of varying degree of fluoroquinolone exposure. The objective of this study was to identify risk factors for the fecal shedding of quinolone-resistant $E$. coli (QREC) from dairy calves and postpartum cows. Rectal swabs from 15 preweaned calves and 5 postpartum cows per farm were collected on 23 Swedish dairy farms to determine the prevalence of QREC. Risk factors for the shedding of QREC were investigated using multivariable statistical models. Quinolone-resistant E. coli were found on all but one farm. Factors associated with QREC shedding by calves were being younger than 18 $\mathrm{d}$, being fed milk from cows treated with antimicrobials, recent use of fluoroquinolones in the herd, carriage of QREC by postpartum cows, and using the calving area never or rarely as a sick pen compared with often. Factors associated with QREC shedding by cows were calving in group pens or freestalls compared with single pens or tiestalls, purchasing cattle, sharing animal transports with other farmers, and poor farm hygiene. Proper biosecurity and improved hygiene, as well as minimizing fluoroquinolone exposure and waste milk feeding, may be important factors to reduce the burden of QREC on dairy farms.

Key words: calf, postpartum cow, quinolone-resistant Escherichia coli, risk factor

Received February 11, 2015.

Accepted May 31, 2015.

${ }^{1}$ Corresponding author: anna.duse@sva.se

\section{INTRODUCTION}

Microorganisms with decreased susceptibility to antimicrobials are one of the greatest threats for human health according to the European Center for Disease Prevention and Control (ECDC, 2007). Special concerns have been raised about the development of resistance to quinolones, which are identified by the World Health Organization (2012) as critically important antimicrobials for human medicine. Results from several surveillance programs in Europe show an increase of some quinolone-resistant pathogens affecting both humans and food-producing animals (EFSA, 2014). In young dairy calves, fluoroquinolone-resistant Escherichia coli have been increasingly involved in bacterial infections (Marchese et al., 2012; Cummings et al., 2014). Like most resistance phenotypes, quinolone-resistant E. coli (QREC) are more common in calves than in older cattle (Kaesbohrer et al., 2012; Swedres-Svarm 2013). The fecal microbiota of calves may therefore serve as a reservoir for QREC, causing intractable infections in cattle. Although QREC is less common in feces from older cattle, studies on adult animals indicate that the shedding of some antimicrobial-resistant E. coli increases around parturition (Watson et al., 2012; Callens et al., 2015). The dam's microbiota might therefore be a reservoir of resistant strains for her offspring (Callens et al., 2015). Hence, it is important to elucidate whether QREC is found in the feces of postpartum cows and what factors affect the shedding of QREC from them.

Knowledge about the emergence and epidemiology of quinolone resistance in fecal $E$. coli from cattle is limited. In our previous study, we identified a few risk factors for the shedding of QREC by calves; one such factor was usage of fluoroquinolones for cows (Duse et al., 2015). Although historic and recent usage of fluoroquinolones has been linked to the presence of QREC (Pereira et al., 2014a; Duse et al., 2015), QREC have also been isolated from animals with no known exposure to quinolones (Taylor et al., 2009; Duse et al., 
2015). These findings indicate that factors other than exposure to fluoroquinolones may be involved in the epidemiology of QREC. We were therefore interested in seeing how farms with high and low prevalence of QREC differed from each other. The objectives of this study were to identify risk factors for the shedding of QREC from preweaned dairy calves and postpartum dairy cows. In particular, we looked at feeding and management factors for preweaned dairy calves. Such knowledge could form the basis for recommendations to reduce the prevalence of QREC on dairy farms.

\section{MATERIALS AND METHODS}

\section{Study Design and Selection of Farms}

We aimed to study differences in management factors on farms where QREC was common and farms where QREC was rare or absent. For this purpose, the study was designed as a case-control study. This study design is useful when the case condition is rare or when time limits extensive sampling (Dohoo et al., 2010). Because historic use of fluoroquinolones and being located in south or east Sweden were found to be significant risk factors for QREC in Duse et al. (2015), we selected only farms matching these criteria to avoid confounding (Dohoo et al., 2010). Moreover, because the farm environment was sampled for a parallel study, we wanted to include farms that were as similar in housing as possible; thus, only farms with insulated freestall barns were eligible for inclusion. Cases and control farms were selected from 84 farms. Based on a previous estimate on the mean within-sample prevalence of fecal QREC for 3 calves on each farm (Duse et al., 2015), we defined cases as farms where the mean within-sample prevalence of QREC exceeded 10\% ( $\mathrm{n}=14$ farms) and controls as those farms where none of the calves had QREC ( $\mathrm{n}=20$ farms). In descending order of QREC prevalence, case farms were asked to take part in the study and 10 farms were recruited; the remaining 4 did not want to participate for various reasons. A total of 11 control farms were asked in random order to participate until 10 farms could be enrolled. Finally, 3 of the 84 farms with moderate mean prevalence of QREC (0.02-0.8\%) were enrolled. These 3 farms did not match the criteria for cases or controls but were selected due to the presence of $E$. coli carrying genes encoding extended-spectrum $\beta$-lactamases (ESBL), which was of interest for a parallel study in which shedding of E. coli resistant to third-generation cephalosporins was studied. Table 1 contains descriptive statistics of the enrolled farms.

\section{Farm Visits}

Each farm was visited once between November 2013 and March 2014. The corresponding author interviewed the farmer or the herdsman regarding herd management, assessed the general hygiene on the farm, and collected fecal samples from calves and postpartum cows. The interview questions were categorized into the following subsets: farm characteristics (number of cattle on the farm, average milk yield, and predominant milking system), veterinary services in the previous 4 mo, management of preweaned dairy calves, management of the calving area and routines around calving, feeding of milk from cows treated with antimicrobials, and biosecurity factors (purchasing cattle, sharing equipment with other livestock farmers, visitor restrictions, and requirements for visitors to use protective clothing). For more details, see Supplemental Tables S1 and S2 (http://dx.doi.org/10.3168/jds.2015-9453). Most questions were semi-closed with space available for the investigator to make notes if alternatives to the options were given. Answers to all questions were given by the herdsman or the farmer, except those about veterinary services, which were compiled from veterinary invoices/records and on-farm records, in cooperation with the respondents. The questionnaire (in Swedish) is available upon request from the corresponding author. Approximate estimates on historic usage of fluoroquinolones in cows and calves from up to $2 \mathrm{yr}$ before the sampling date were obtained from our previous study (Duse et al., 2015). The interview did not include questions that could interfere with the personal integrity of the farmers and hence, an ethical approval for research on humans was not deemed necessary, according to the act on ethical review of research involving humans (SFS 2003:460) in the Swedish Code of Statutes. The majority of the questions in the interview were tested on 6 other farmers before the study and no corrections had to be made to the questionnaire.

To assess hygiene on the farm, specific locations (group calf pens, single calf pens, feed and water troughs in calf pens, young stock area, calving area, dry cow area, lactating cow area, and milk feeding equipment) were scored on predefined scales of 0 to 3 , where 0 was cleanest and 3 dirtiest (Supplemental Table S3; http://dx.doi.org/10.3168/jds.2015-9453). A mean hygienic score for each farm was calculated as the sum of all scores divided by the number of locations evaluated.

Fecal samples were collected rectally with Amie's charcoal culture swabs (Copan Diagnostics Inc., Murrieta, CA) from up to 15 calves and up to 5 cows in each herd. The samples were immediately mailed at 
RISK FACTORS FOR QUINOLONE-RESISTANT ESCHERICHIA COLI

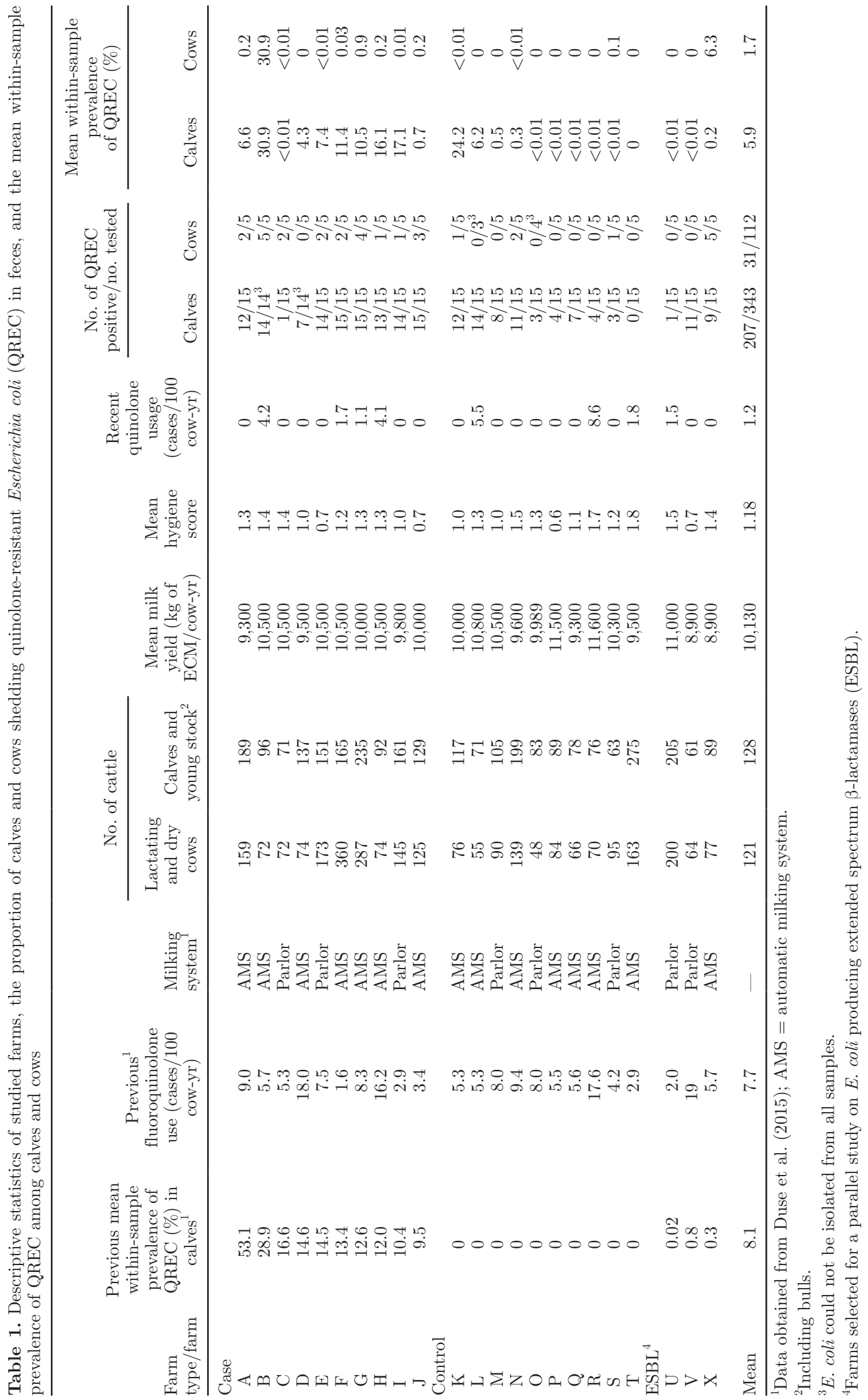


ambient temperature to the National Veterinary Institute (SVA, Uppsala, Sweden). Sampled calves were aged 0 to $30 \mathrm{~d}$, were visually healthy, and had never received treatment with antimicrobials since birth. If fewer than 15 calves on the farm were in the desired age span, all calves were sampled. If more than 15 calves for sampling were in the correct age span, calves were spread out evenly by age at each farm. If 2 or more calves were of the same age, 1 of those calves was selected at random. Information was collected about the calves' identification number, sex, age, housing, and whether they had received either colostrum from cows treated with antimicrobials at drying off (waste colostrum, WC) or milk from cows treated with antimicrobials during lactation (waste milk, WM) or no such colostrum or milk. Cows eligible for sampling were 0 to $3 \mathrm{~d}$ postpartum, were visually healthy, and had not been treated with antimicrobials from calving and up to sampling. Both primi- and multiparous cows were sampled. None of the farms had more than 5 cows that were 0 to $3 \mathrm{~d}$ postpartum, and thus all cows that were in this postpartum time span had to be sampled on each farm. The following information was collected: identification number, DIM at sampling, days spent in calving area, housing type at calving, treatment with antimicrobials at drying off, and whether the calving was assisted or not. If there were fewer than 15 calves and 5 postpartum cows available at the visit, sampling material was left for supplementary collection of cow and calf fecal samples by the farmer. Farmer sampling of feces from calves was started approximately 1 mo after the visit to minimize the risk of sampling the same calf twice. Samples from postpartum cows were collected whenever a calving occurred. These additional samples were mailed at ambient temperature to SVA. Farmers were encouraged to send samples on Sundays to Thursdays to ensure delivery before the weekend. Because all animals in the study were privately owned and no invasive procedures were done, ethical approval for the use of laboratory animals was not needed according to the Board of Agriculture's regulations and general advice on laboratory animals (SJVFS 2012:26).

\section{Sample Analyses}

Nalidixic acid resistance is often regarded as a generic marker for quinolone resistance in gram-negative organisms (Ruiz, 2003). In short, the percentage of $E$. coli resistant to nalidixic acid was determined by plating each fecal sample on Petrifilm Select $E$. coli Count (SEC) plates (3M Microbiology Products, St. Paul., MN) with and without nalidixic acid. The SEC plate is a culture medium consisting of plastic film with grids coated with selective agents, nutrients, and gelling agent. The indicator $\beta$-glucuronidase is incorporated in the gel for detection of $E$. coli, which eliminates the need for colony identification because only $E$. coli is conspicuous on SEC plates (Frampton and Restaino, 1993). The within-sample prevalence of QREC was determined with a method modified from $\mathrm{Wu}$ et al. (2008). The selective properties of the SEC plates with nalidixic acid had been evaluated in a pilot study by parallel plating a subset of the fecal samples on SEC and MacConkey agar plates supplemented with $32 \mathrm{mg} / \mathrm{L}$ nalidixic acid. Both SEC and MacConkey agar plates yielded similar counts (A. Duse, unpublished data). We have previously validated MacConkey plates (with and without nalidixic acid) for within-sample prevalence of QREC (Duse et al., 2015).

The fecal samples were analyzed within 24 to $48 \mathrm{~h}$ after collection. Upon arrival at the laboratory, each rectal swab was vortexed in $3 \mathrm{~mL}$ of $0.9 \%$ saline to release fecal content. This fecal suspension constituted the nondiluted suspension $\left(10^{\circ}\right)$ from which 10-fold dilutions down to $10^{-6}$ were made in $0.9 \%$ saline to determine the counts (cfu) of $E$. coli from the suspensions. The total count of $E$. coli was determined as follows. One-milliliter aliquots of dilutions $10^{-4}$ and $10^{-6}$ (for calf samples) and $10^{-3}$ and $10^{-5}$ (for cow samples) (suitable dilutions determined in the pilot study; A. Duse, unpublished data) were placed onto the bottom film of an SEC plate. The bottom film was then covered with the top film and the plate set aside to allow the liquid to be absorbed into the medium.

The counts of nalidixic acid-resistant E. coli were determined as follows. A stock solution of nalidixic acid sodium salt at $672 \mu \mathrm{g} / \mathrm{mL}$ (Sigma-Aldrich Co., St. Louis, MO) was prepared. Fifty microliters of stock solution was added to $1,000 \mu \mathrm{L}$ of the fecal sample dilutions of $10^{0}$ and $10^{-2}$ to obtain a final concentration of $32 \mu \mathrm{g} /$ $\mathrm{mL}$ nalidixic acid. The entire volume of this suspension $(1,050 \mu \mathrm{L})$ was plated onto SEC plates as described above. Quality control was conducted for each batch of nalidixic acid stock solution, using E. coli ATCC 25922 as a susceptible reference strain and E. coli 4.7 (European Union Reference Laboratory-Antimicrobial Resistance) as a QREC reference strain.

Plates were incubated for 18 to $24 \mathrm{~h}$ at $42^{\circ} \mathrm{C}$, and dark green to light blue-green colonies were counted. The $E$. coli colonies on SEC plates with nalidixic acid were considered to be QREC. The total number of E. coli colonies was determined from plates without nalidixic acid. To determine the within-sample prevalence $(\%)$ of QREC in each sample, the number of resistant E. coli was divided by the total number of $E$. coli colonies and multiplied by 100 . 


\section{Statistical Analysis}

Data were entered in Access or Excel spreadsheets (Microsoft Corporation, Seattle, WA) and exported into Stata 13 (release 13.1, StataCorp., College Station, TX). Descriptive statistics on the prevalence of animals shedding QREC and the mean within-sample prevalence of QREC were calculated. The prevalence in cows and calves was compared using Fisher's exact test for the proportion of positive animals and Wilcoxon rank sum test for the within-sample prevalence, respectively. Questionnaire data were compiled manually; responses were grouped into as few categories as possible (Supplemental Tables S1 and S2; http://dx.doi.org/10.3168/ jds.2015-9453). Categorization was done based on biological plausibility or to yield groups of equal size (Supplemental Tables S1 and S2).

In the respective risk factor analyses, the study units were the calf and the cow. The univariable association of potential predictor variables (Supplemental Table S1; http://dx.doi.org/10.3168/jds.2015-9453) to the within-sample prevalence of QREC among calves was investigated using zero-inflated negative binomial regression with the ZINB function in Stata. Few cows had a high within-sample prevalence of QREC; therefore, using the within-sample prevalence as an outcome for cows would have led to an overestimation of the importance of risk factors associated with these particular cows. Hence, only risk factors for being QREC-positive or not were sought. The univariable associations of potential predictor variables (Supplemental Table S2; http://dx.doi.org/10.3168/jds.2015-9453) with the probability of being QREC-positive in cows and calves were investigated using logistic regression with the LOGIT function in Stata. In both the univariable zero-inflated negative binomial and logistic regression analyses, a robust variance estimator was used to account for the clustering of observations at the herd level. The assumption of linearity between continuous predictor variables and outcome variables was checked visually with the LINTREND function in Stata. Continuous variables not linearly related to the outcome variables were categorized using biologically important cut-off values or cut-off values that resulted in evensized groups.

Zero-inflated negative binomial (calves) and logistic (cows) multivariable regression models were developed with robust standard errors to account for clustering within herds. The zero-inflated negative binomial regression model for risk factors in calves included the negative binomial part (which models the withinsample prevalence of QREC in the sample) and the logistic regression part (which models the probability of isolating zero QREC in the sample). For simplicity, the results are shown in Table 2 as the probability of being QREC-positive instead of being QREC-negative. A manual, stepwise-forward selection process was used to build multivariable models by testing all variables in both the logistic and the negative binomial parts of the model. Variables were tested in a descending order of significance, starting with the variable with the lowest $P$-value from the univariable analyses. The stepwise-forward procedure is an alternative to stepwisebackward elimination and is more suitable for small data sets with many potential predictor variables where it is impossible to fit the maximum model (Dohoo et al., 2010). Collinearity was assessed by pair-wise correlation using Spearman rank-order correlations. All biologically plausible predictor variables were tested in the models provided that there was no collinearity ( $\mathrm{r}$ $>|0.7|$ ). Only the most significant variable (univariable analyses) of collinear variables was tested in the model. Potential confounders were defined as variables that changed the coefficient for one or more variable by more than $30 \%$. All variables with a $P$-value $\leq 0.05$ were retained in the final model. All possible 2-way interactions between retained variables were tested, but none of them were significant. Model fit was investigated by generating and comparing expected probabilities to observed probabilities for both models. For the logistic regression model, fit was also assessed using the Hosmer-Lemeshow $\chi^{2}$-test with the data portioned in 10 deciles. The effect of excluding observations with missing data was analyzed by comparing coefficients and fit-to-models where these observations were included. If coefficients and model fit were indifferent in these 2 models, observations with missing data were excluded; otherwise they were retained in the final model.

\section{RESULTS}

\section{Descriptive Statistics of Calves and Cows}

Fecal samples were collected from 349 calves and 119 cows on 23 farms. Three of the calves from 1 farm were older than $30 \mathrm{~d}$ at the time of sampling and were therefore excluded from the study. On 1 farm, 16 instead of 15 calves were sampled, and on 4 farms, 6 instead of 5 cows were sampled. To ensure that no more than 15 calves and 5 cows on each farm were used in the data analyses, the most recently collected sample from these farms was excluded. Hence, samples from 345 calves and 115 cows were analyzed for the within-sample prevalence of QREC.

The median age of the sampled calves was $12 \mathrm{~d}$ (25th percentile, $6 \mathrm{~d}$; 75 th percentile, $20 \mathrm{~d}$ ). The calves were housed in single pens (53\%), group pens (41\%), with their own dam (5\%), or with nursing cows (1\%). Thirty 
Table 2. Results from the final multivariable zero-inflated negative binomial regression model for factors associated with the shedding of quinolone-resistant Escherichia coli (QREC) in fecal samples from preweaned calves 0 to $30 \mathrm{~d}$ of age $(\mathrm{n}=337)$

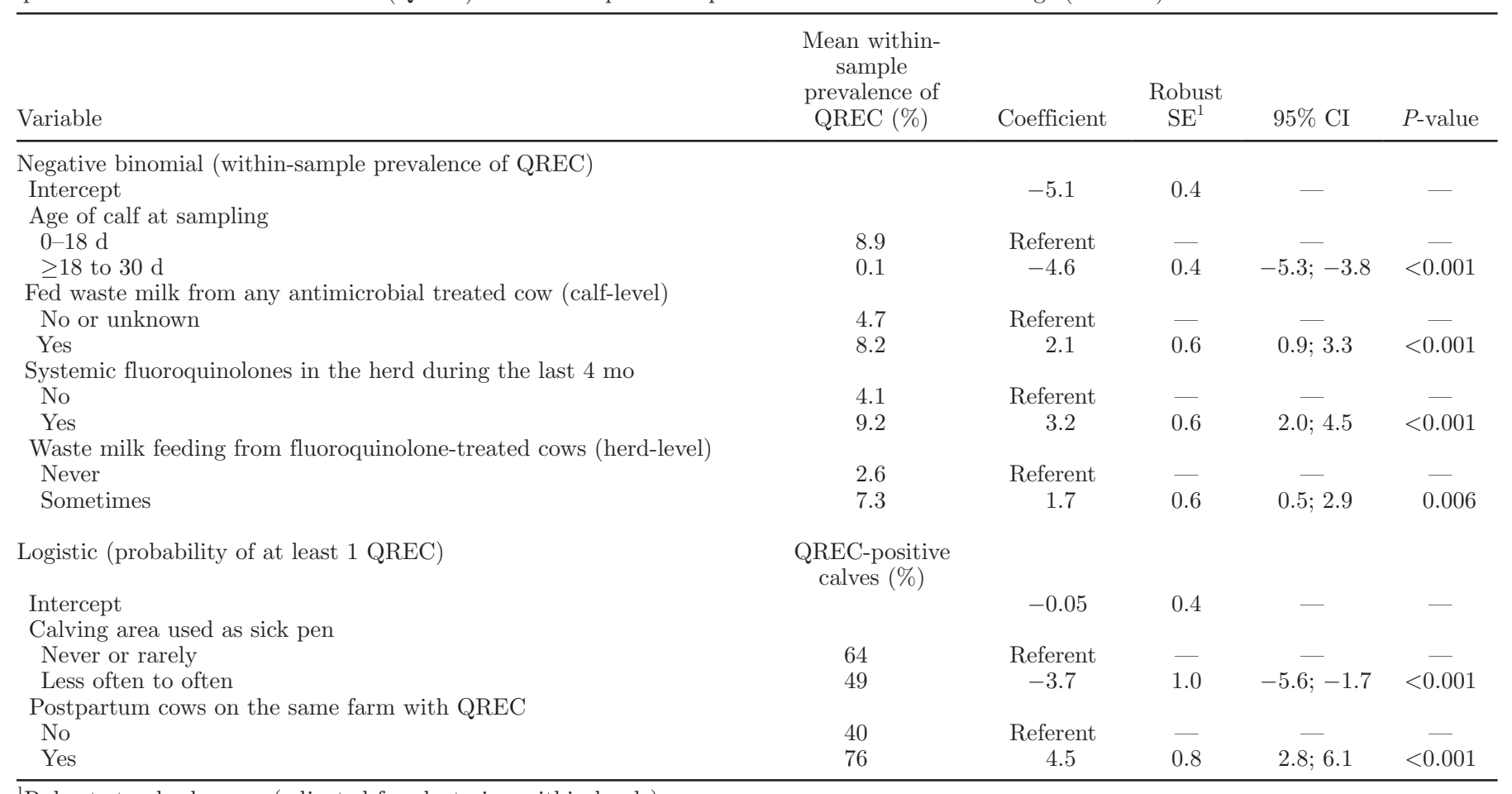

${ }^{1}$ Robust standard errors (adjusted for clustering within herds).

percent of the calves had been given colostrum from cows treated with antimicrobials at drying off and $68 \%$ of the calves had been fed colostrum from cows that were not treated; for the remaining $2 \%$ of the calves, the farmer was uncertain whether the calf had received colostrum from a treated cow. Thirty-three percent of the sampled calves had been fed WM, $56 \%$ had not, and the farmer was unsure for the remaining $11 \%$ of the calves. Supplemental Table S1 (http://dx.doi. org/10.3168/jds.2015-9453) contains additional descriptive statistics for the calves.

The median DIM for the sampled cows was $1 \mathrm{~d}$ (25th percentile, $0 \mathrm{~d}$; 75 th percentile, $2 \mathrm{~d}$ ). The cows calved mostly in single pens (46\%), followed by group pens $(28 \%)$, tiestalls $(14 \%)$, and other calving facilities (11\%). The majority of cows calved unassisted (85\%). The cows were moved to the calving area at 0 to $59 \mathrm{~d}$ before calving, with a median of $2.5 \mathrm{~d}$ (25th percentile, $1 \mathrm{~d}$; 75th percentile, $10 \mathrm{~d}$ ). Supplemental Table S2 (http://dx.doi.org/10.3168/jds.2015-9453) contains additional descriptive statistics for cows.

\section{Occurrence of Fecal QREC in Calves and Postpartum Cows}

Growth of $E$. coli was observed in all but 2 fecal samples from calves and in all but 3 fecal samples from cows (Table 1). Hence, further analyses were conducted on samples from 343 calves and 112 cows. Quinoloneresistant $E$. coli could be detected in significantly $(P<$ $0.001)$ more calf $(60 \%)$ than cow $(27 \%)$ fecal samples. Likewise, a significantly $(P<0.001)$ higher within-sample prevalence was found in feces from calves than in that from cows. The median within-sample prevalence of QREC in calves was $0.0003 \%$ (25th percentile, $0 \%$; 75th percentile, $0.7 \%$ ), ranging between 0 and $100 \%$ for individual calves. The median within-sample prevalence of QREC in cows was $0 \%$ (25\% percentile, $0 \%$; $75 \%$ percentile, $0.0006 \%$ ), ranging between 0 and $100 \%$ for individual cows. Quinolone-resistant E. coli were isolated from calves on all but one farm and from cows on 13 of 23 farms. The mean within-sample prevalence of QREC for individual farms ranged from 0 to $30.9 \%$ for both calves and cows (Table 1).

\section{Univariable Association of Potential Risk Factors with Shedding of Fecal QREC}

The univariable analyses of potential risk factors for the shedding of QREC from calves and cows are presented in Supplemental Tables S1 and S2 (http:// dx.doi.org/10.3168/jds.2015-9453). For the withinsample prevalence of QREC in calves, significant $(P$ $<0.05)$ associations were found with variables in all 
subsets. The only variable significantly associated with calves carrying at least one QREC was the variable "at least 1 postpartum cow with QREC on the farm." For cows carrying QREC, significant univariable associations were only found in the subsets "biosecurity" and "cow" factors.

There was a high degree of collinearity between the variables containing information about whether milk from cows treated with different antimicrobials was fed to calves $(\mathrm{r}=0.62-0.90)$. Hence, only the most significant variables were tested in the multivariable analyses (Supplemental Table S1; http://dx.doi. org/10.3168/jds.2015-9453). These were "feeding milk from fluoroquinolone-treated cows" for the outcome within-sample prevalence and "feeding milk from benzyl penicillin-treated cows" for the outcome proportion of QREC-positive calves.

\section{Risk Factors for Shedding of Fecal QREC from Calves 0 to 30 Days Old}

The multivariable analysis (Table 2) showed that calves $18 \mathrm{~d}$ or older had significantly lower withinsample prevalence of QREC than calves aged 0 to 17 d. Plotting the within-sample prevalence of QREC as a function of calf age revealed a clear decline in the within-sample prevalence of QREC at $18 \mathrm{~d}$, justifying the grouping into 2 categories ( 0 to $17 \mathrm{~d}$ and $18 \mathrm{~d}$ and older, Figure 1). Use of fluoroquinolones in the previous $4 \mathrm{mo}$ in any cattle category on the farm significantly increased the proportion of QREC shed by calves. Feeding milk from cows treated with fluoroquinolones (farm level) also resulted in a higher proportion of resistant $E$. coli in feces from calves. Individual calves fed WM (here defined as milk from antimicrobial-treated cows) had a higher within-sample prevalence of QREC than calves not fed such milk or with unknown status. Figure 2 describes the within-sample prevalence for calves fed or not fed WM as a function of calf age. Calves fed WM had a higher proportion of QREC directly after birth compared with those never fed WM, but the prevalence decreased to the level of calves not fed WM at around $20 \mathrm{~d}$. Calves on farms where at least one of the postpartum cows was positive for QREC were more likely to carry QREC than calves on farms where all sampled postpartum cows were negative for QREC. Finally, the probability of isolating at least one QREC was higher when the calving area was never or rarely used as a sick pen than when it was often used as such.

\section{Risk Factors for Shedding of QREC in Feces from Postpartum Cows}

Cows that were housed in group pens or freestalls around parturition were more likely to shed QREC than cows in single pens or tiestalls (Table 3). There were greater odds of QREC in cows from farms that occasionally purchased new cattle than from farms that never purchased any. Sharing animal transport with other livestock farmers was also associated with greater

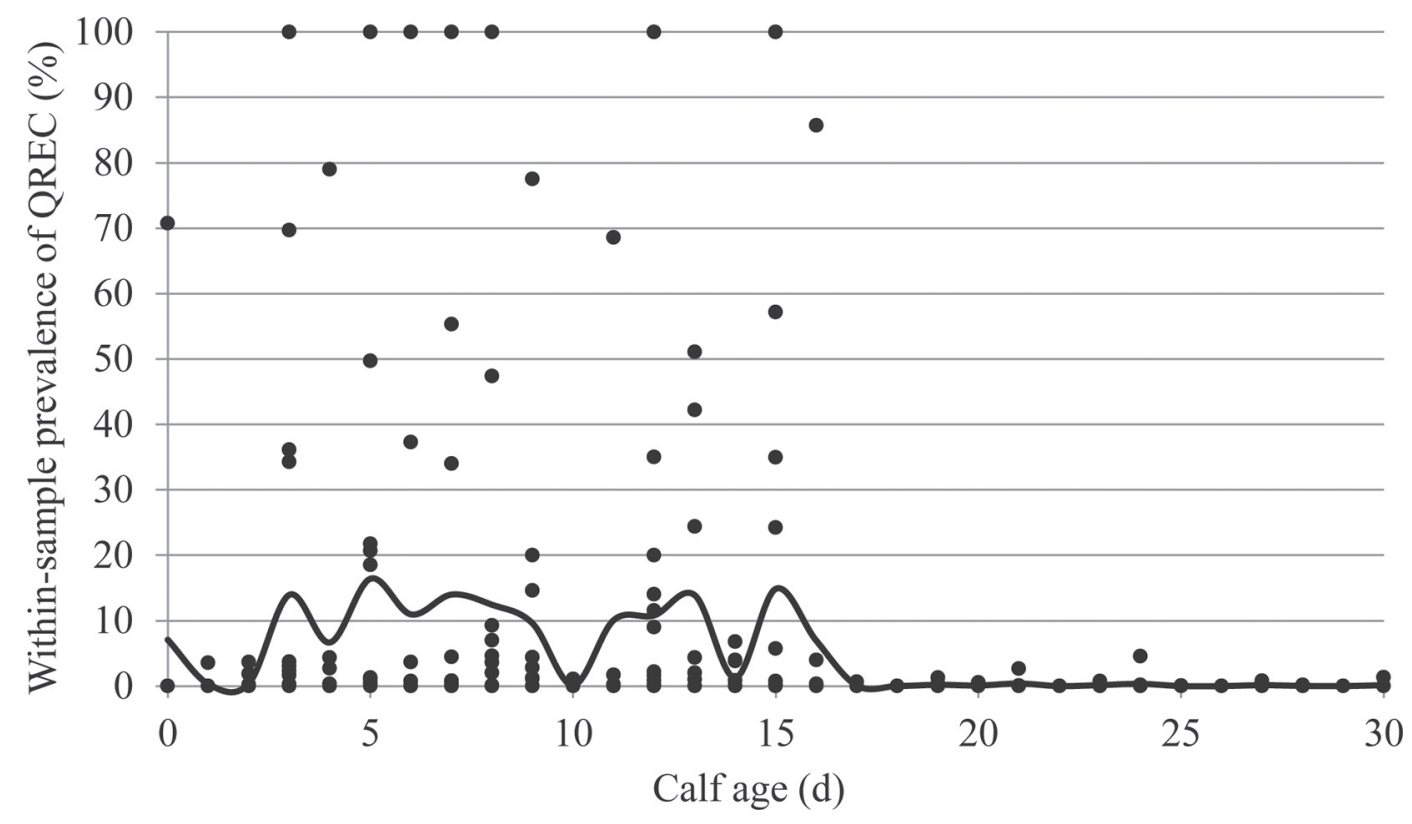

Figure 1. The within-sample prevalence of quinolone-resistant Escherichia coli (QREC) from calves (circles for individuals and solid line for the age-mean) as a function of calf age. 
6394

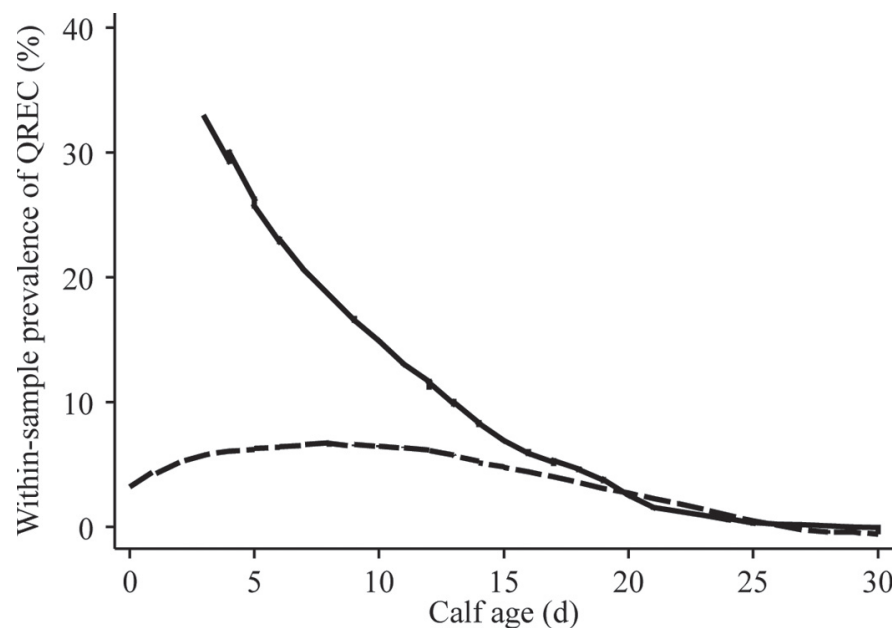

Figure 2. Lowess smoother (bandwidth 0.8) for the mean withinsample prevalence of quinolone-resistant Escherichia coli (QREC) in calves fed waste milk from cows treated with antimicrobials during lactation (solid line) and calves not fed such milk (dashed line) as a function of calf age.

odds of carrying QREC. Finally, cows from farms with a below-average hygiene score were more likely to have QREC than those from farms with higher than average hygiene score.

\section{DISCUSSION}

Risk factors for the shedding of QREC by cattle have not been extensively studied. To our knowledge, limited information is available on the association between farm management-related factors and the prevalence of QREC in preweaned calves and postpartum cows. In line with other reports, QREC was more often isolated from preweaned calves than from mature cows after calving (Swedres-Svarm 2013). Age of the calf was the most important risk factor for fecal shedding of QREC in this study. Age-related decline in the shedding of resistant E. coli by calves is a well-established phenomenon and seems to be consistent for different resistance phenotypes (Hoyle et al., 2004; de Verdier et al., 2012; Duse et al., 2015). However, the age-dependent decline in this study was not linear. Instead, we observed a sudden and significant reduction in the relative number of QREC around $18 \mathrm{~d}$ of age. This sudden decline was conspicuous and cannot be explained within the scope of this study. Nevertheless, factors related to the immune system or the establishment of the gastrointestinal (GI) microbiota in the calves are probably involved. The calf GI microbiota evolves rapidly during its first weeks (Mackie et al., 1999; Oikonomou et al., 2013; Klein-Jöbstl et al., 2014). The succession of the GI microbiota depends on numerous internal factors, such as immunology and microbial interactions (Mackie et al., 1999), and various species or strains may be more or less adapted to the specific conditions of the intestinal environment at different ages (Khachatryan et al., 2004). The sudden decline in QREC may therefore be due to changed conditions in the GI tract and may occur as part of the normal succession and turnover of strains during the first weeks of life. The question remains, however, why QREC seem to be more adapted to the intestines of calves less than $18 \mathrm{~d}$ old.

In addition to age, other factors affected the shedding of QREC by calves. Use of systemic fluoroquinolones in the herd during the previous 4 mo increased the within-sample prevalence of QREC in the feces of calves but did not increase the odds of cows and calves being QREC-positive. Thus, although recent exposure to quinolone antimicrobials in the herd seems

Table 3. Results from the final multivariable logistic regression model for factors associated with the shedding of quinolone-resistant Escherichia coli (QREC) in fecal samples from cows 0 to $3 \mathrm{~d}$ after calving $(\mathrm{n}=111)$

\begin{tabular}{|c|c|c|c|c|c|c|}
\hline Variable & $\begin{array}{l}\text { QREC-positive } \\
\text { cows }(\%)\end{array}$ & Coefficient & $\begin{array}{l}\text { Robust } \\
\mathrm{SE}^{1}\end{array}$ & $\mathrm{OR}^{2}$ & $95 \% \mathrm{CI}$ & $P$-value \\
\hline Intercept & & 0.005 & 0.005 & - & - & - \\
\hline Group pens or freestall cubicles & 48 & 2.1 & 0.7 & 7.9 & $2.2 ; 28.7$ & 0.002 \\
\hline \multicolumn{7}{|l|}{ Overall farm hygiene } \\
\hline Better than average & 18 & Referent & - & 1 & - & - \\
\hline No & 20 & Referent & - & 1 & - & - \\
\hline Yes & 42 & 1.9 & 0.6 & 6.9 & $2.1 ; 22.9$ & 0.002 \\
\hline \multicolumn{7}{|c|}{ Sharing animal transport with other livestock farmers } \\
\hline No & 16 & Referent & - & 1 & - & — \\
\hline Yes & 44 & 1.8 & 0.6 & 6.0 & $1.9 ; 18.8$ & 0.002 \\
\hline
\end{tabular}

${ }^{1}$ Robust standard errors (adjusted for clustering within herds).

${ }^{2}$ Odds ratio. 
to favor already existing populations of QREC, it does not seem to determine whether they become QRECpositive or not. However, we used only a qualitative variable for fluoroquinolone use because this drug was used rarely during the 4 mo. If we had instead assessed the prevalence of QREC in relation to the number of fluoroquinolone treatments, the number of quinolone treatments might have been associated with the number of QREC-positive individuals. Enrofloxacin is the only quinolone drug licensed for cattle in Sweden and was the quinolone used on farms in the present study. Pereira et al. (2014a) observed that calves that had been treated at least once with enrofloxacin had more QREC isolates than calves that had not received enrofloxacin. Likewise, Bosman et al. (2014) showed higher odds of QREC in veal calves on farms that had used more enrofloxacin than the median farm in the study compared with farms that had used less than the median farm. Systemic treatment with enrofloxacin is likely to result in low concentrations of its metabolite ciprofloxacin in the intestinal content, as shown in pigs (Wiuff et al., 2002). We assume that low concentrations of ciprofloxacin may be sufficient to enrich the QREC population in the gut, based on the results of Gullberg et al. (2011). All quinolone antimicrobials inhibit the activity of DNA gyrase and topoisomerase IV, enzymes that relax the supercoiling of bacterial DNA and complete cell division (Ruiz, 2003). Resistance in E. coli is often a result of mutations that modify the DNA gyrase or topoisomerase IV binding sites, which may affect the susceptibility of all quinolones (Ruiz, 2003). Hence, the use of one quinolone (such as enrofloxacin) may select for resistance to another quinolone (such as nalidixic acid). On one of the farms (farm T), we were unable to isolate any QREC although the farmer reported both recent and historic usage of fluoroquinolones. Inability to find QREC isolates could be due to the limited number of animals sampled or imprecision of the methods used for culturing, but it could also be due to freedom from QREC on this farm. However, proving freedom from QREC would necessitate sampling of the majority of the animals in the herd and even this would not fully answer the question.

Feeding WM from cows treated with fluoroquinolones to calves (herd level) increased the within-sample prevalence of QREC in the calf feces, as in our previous study (Duse et al., 2015). However, the high degree of collinearity between all variables about WM feeding (from cows treated with benzylpenicillin, fluoroquinolones, tetracycline, or trimethoprim-sulfonamide combinations) indicates that farmers had similar routines for the feeding of WM independent of what type of antimicrobial residue it may contain. It is therefore likely that the increased within-sample prevalence of QREC is not solely a result of feeding WM from fluoroquinolone-treated cows but rather a result of WM feeding in general. Likewise, on the individual calf level, feeding WM regardless of the type of antimicrobial treatment the cow was given led to an increased proportion of QREC in the calf feces. Likewise, WM feeding has been shown by others to increase the shedding of resistant E. coli in feces of calves (Wray et al., 1990; Aust et al., 2013; Brunton et al., 2014; Pereira et al., 2014b; Rebelo, 2014) and when the concentration of antimicrobials in WM is low (Pereira et al., 2014b). The use of fluoroquinolones during the previous 4 mo was low (only 1.2 cases per 100 cow-years) and hence, few of the calves in the current study could have been fed WM from fluoroquinolone-treated cows. Instead, most calves were probably fed WM from cows treated with benzyl penicillin (21 cases per 100 cow-years during the previous 4 mo; results not shown). Although E. coli is intrinsically resistant to penicillin, at least one hypothesis can be proposed as to why feeding WM with penicillin would increase the within-sample prevalence of QREC. The GI microbiota of newborn calves contains many penicillin-susceptible species (Mayer et al., 2012; Oikonomou et al., 2013; Klein-Jöbstl et al., 2014). Exposure to penicillin in milk may therefore result in clearance of these penicillin-susceptible bacteria from the GI tract, thereby making it easier for QREC to establish. In general, the GI microbiota in calves increases markedly in diversity and species richness after 2 to $3 \mathrm{wk}$ of age (Oikonomou et al., 2013; Klein-Jöbstl et al., 2014). We assume therefore that the GI microbiota in calves less than 2 to 3 wk old may be more vulnerable to antimicrobial exposure than the more established microbiota in calves older than 2 to 3 wk of age. Hence, according to Figure 2, feeding WM seems to enrich the QREC population predominantly in calves less than 2 to $3 \mathrm{wk}$ old, but this is only speculative and warrants further study. In Duse et al. (2015), we found a significant interaction between WM feeding and fluoroquinolone use in cows. Such an interaction was not found in the present study and neither were these variables correlated. We can assume, however, that feeding WM from fluoroquinolone-treated cows would not only disturb the GI microbiota, which increases the risk of calves being colonized with QREC, but also directly favor any QREC that has colonized the tract.

Breaches in farm biosecurity (e.g., purchasing new cattle and sharing animal transports with other farmers) were associated with higher odds of shedding QREC by cows. Taylor et al. (2009) concluded that QREC may spread between farms and that some biosecurity breaches were associated with increased odds of fluoroquinolone-resistant E. coli and Campylobacter. Escherichia coli can survive for a long period outside 
the intestinal tract of mammals (Sjögren, 1995). In addition, mutations conferring resistance to quinolones are not always associated with a fitness cost for the bacteria; instead, fitness may actually increase (de Lastours et al., 2014; Machuca et al., 2014). It is therefore logical to assume that QREC may be introduced to, and established in, naïve farm animals through purchase of animals carrying resistant bacteria or through contaminated farm equipment. A recent report on clonal dissemination of fluoroquinolone-resistant $E$. coli among calves on different farms in Italy (Marchese et al., 2012) supports the theory that QREC could be transmitted between farms. Factors affecting the spread of QREC may therefore be similar to those associated with the transmission of any bacterial agent. Based on these results, we are currently investigating the clonality of QREC strains on and between farms in this study.

Both poor farm hygiene and group-pen calving were associated with increased odds of fecal shedding of QREC by cows. In the study by Taylor et al. (2009), hygiene status had no influence on the presence of QREC in the feces of pigs. The inconsistency between the studies may be a result of subjectivity in the hygiene rating. Nevertheless, it can be assumed that the on-farm epidemiology of QREC is similar to that of other fecal-oral transmitted bacteria (e.g., the extensively studied Mycobacterium avium ssp. paratuberculosis). Increased transmission of $M$. avium ssp. paratuberculosis has been associated with poor hygiene (Johnson-Ifearulundu and Kaneene, 1998) and grouppen calving (Pithua et al., 2013). In addition, it is likely that heavily feces-contaminated farms offer perfect conditions for the spread of fecal-oral transmitted bacteria, such as QREC. Bosman et al. (2014) also found that poor farmworker hygiene (e.g., changing clothes infrequently) increases the occurrence of fluoroquinoloneresistant $E$. coli in veal calves. Unexpectedly, calves in this study were more likely to carry QREC on farms that rarely used the calving pen as a sick pen compared with often. At least in Sweden, where prophylactic use of antimicrobials is not recommended, it is more likely that sick cows are exposed to antimicrobials (with subsequent shedding of resistant $E$. coli) than healthy cows. On the other hand, the farms that often used the calving pen as sick pen (67\%) tended to wet-clean it more often than the farms that rarely used it as a sick pen (41\%; results not shown). Frequent use of the calving pen as sick pen may therefore be a proxy for frequent cleaning of the calving pen, which is likely to reduce the risk for dissemination of QREC. Similarly, cleaning of the maternity pen decreased the odds of a herd being positive for M. avium ssp. paratuberculosis (Johnson-Ifearulundu and Kaneene, 1998).
Finally, calves were more likely to shed QREC on farms with at least one QREC positive cow. This indicates that QREC shedding in one age category may be important for QREC shedding in other age categories on the farm. In 7 of the calves (Figure 1), the within-sample prevalence was $100 \%$, meaning that all E. coli isolates in the sample suspension were QREC. This is an important finding because these calves may efficiently increase the ratio of QREC to susceptible E. coli in the farm environment, which in turn may increase the risk for other individuals to be colonized with QREC. Therefore, we assume that when the prevalence of QREC is high in calves, these bacteria would be more common in the farm environment and in other age categories than when the prevalence of QREC is low among calves. This hypothesis is currently being investigated in an ongoing study, where the genetic diversity of QREC is also being determined to investigate the dissemination of QREC on dairy farms.

The present study has some limitations. The study design (case-control) was chosen to ensure that the variation in the prevalence of QREC was large enough to identify differences in management routines. This was done at the expense of the external validity of the results because the farms included were not chosen by random nor were they representative of a larger population; thus, care should be taken when extrapolating the results. The small sample size may have resulted in type II errors; that is, failing to detect factors that actually are associated with QREC shedding, but the limited availability of case farms reduced the sample size. Finally, the animals selected for sampling were not selected completely at random, which could interfere with the internal validity of the results. However, no differences in age, sex, or DIM were observed when comparing calves and cows, respectively, on case and control farms. In addition, farmer sampling may have introduced bias in the results; for example, by sampling antimicrobial-treated or unhealthy calves or by providing faulty submission forms. By providing the farmers with clear sampling instructions, we tried to minimize such errors. Management routines at the time of farmer sampling could also have been different from those present at the farm visit, but we assume that such short-term changes would have a negligible effect on the occurrence of QREC. Moreover, we used categorized variables in the analyses and it is possible that the categorization used was not the most appropriate and that we, by testing only one type of categorization, could have overlooked factors of importance. Despite these limitations, we assume that our results give a valid indication of factors involved in the epidemiology of QREC. 


\section{CONCLUSIONS}

The prevalence of QREC was higher in calves younger than $18 \mathrm{~d}$ of age compared with calves 18 to $30 \mathrm{~d}$ of age. More calves were QREC positive if at least one postpartum cow was QREC positive, indicating that QREC spreads between age categories. In addition, exposure to fluoroquinolones as well as feeding WM increased the within-sample prevalence of QREC. For postpartum cows, biosecurity breaches (purchasing new animals and sharing animal transports with other farms), poor hygiene, and group-pen calvings were associated with increased odds of shedding QREC. Proper biosecurity and improved hygiene, as well as minimizing fluoroquinolone treatment and WM feeding, may be important factors to reduce the burden of QREC on dairy farms.

\section{ACKNOWLEDGMENTS}

The Swedish Farmers' Foundation for Agricultural Research (Stockholm, Sweden) is gratefully acknowledged for financing the study (grant number V1230008). Participating farmers are acknowledged for welcoming us on the farms and for contributing with samples and questionnaire data. The authors also acknowledge Maria Persson (National Veterinary Institute, Uppsala, Sweden) for excellent laboratory work.

\section{REFERENCES}

Aust, V., K. Knappstein, H.-J. Kunz, H. Kaspar, J. Wallmann, and M. Kaske. 2013. Feeding untreated and pasteurized waste milk and bulk milk to calves: Effects on calf performance, health status and antibiotic resistance of faecal bacteria. J. Anim. Physiol. Anim. Nutr. (Berl.) http://dx.doi.org/10.1111/jpn.12019.

Bosman, A. B., J. A. Wagenaar, J. A. Stegeman, J. C. M. Vernooij, and D. J. Mevius. 2014. Antimicrobial resistance in commensal Escherichia coli in veal calves is associated with antimicrobial drug use. Epidemiol. Infect. 142:1893-1904. http://dx.doi. org/10.1017/S0950268813002665.

Brunton, L. A., H. E. Reeves, L. C. Snow, and J. R. Jones. 2014. A longitudinal field trial assessing the impact of feeding waste milk containing antibiotic residues on the prevalence of ESBL-producing Escherichia coli in calves. Prev. Vet. Med. http://dx.doi. org/10.1016/j.prevetmed.2014.08.005.

Callens, B., C. Faes, D. Maes, B. Catry, F. Boyen, D. Francoys, E. de Jong, F. Haesebrouck, and J. Dewulf. 2015. Presence of antimicrobial resistance and antimicrobial use in sows are risk factors for antimicrobial resistance in their offspring. Microb. Drug Resist. 21:50-58. http://dx.doi.org/10.1089/mdr.2014.0037.

Cummings, K. J., V. A. Aprea, and C. Altier. 2014. Antimicrobial resistance trends among Escherichia coli isolates obtained from dairy cattle in the northeastern United States, 2004-2011. Foodborne Pathog. Dis. 11:61-67. http://dx.doi.org/10.1089/fpd.2013.1605.

de Verdier, K., A. Nyman, C. Greko, and B. Bengtsson. 2012. Antimicrobial resistance and virulence factors in Escherichia coli from Swedish dairy calves. Acta Vet. Scand. 54:2 http://dx.doi. org/10.1186/1751-0147-54-2.

de Lastours, V., A. Bleibtreu, F. Chau, C. Burdet, X. Duval, E. Denamur, and B. Fantin. 2014. Quinolone-resistant Escherichia coli from the faecal microbiota of healthy volunteers after ciprofloxacin exposure are highly adapted to a commensal lifestyle. J. Antimicrob. Chemother. 69:761-768. http://dx.doi.org/10.1093/jac/ dkt422.

Dohoo, I., W. Martin, and H. Stryhn. 2010. Veterinary Epidemiologic Research. 2nd ed. VER Inc., Charlottetown, Prince Edward Island, Canada.

Duse, A., K. P. Waller, U. Emanuelson, H. E. Unnerstad, Y. Persson, and B. Bengtsson. 2015. Risk factors for antimicrobial resistance in fecal Escherichia coli from preweaned dairy calves. J. Dairy Sci. 98:500-516. http://dx.doi.org/10.3168/jds.2014-8432.

ECDC. 2007. Annual epidemiological report on communicable disease in Europe. European Centre for Disease Prevention and Control, Stockholm, Sweden.

European Food Safety Authority (EFSA). 2014. Scientific Report of EFSA: EU summary report on antimicrobial resistance in zoonotic and indicator bacteria from humans, animals and food 2012. EFSA J. 12:336.

Frampton, E. W., and L. Restaino. 1993. Methods for Escherichia coli identification in food, water and clinical samples based on betaglucuronidase detection. J. Appl. Bacteriol. 74:223-233. http:// dx.doi.org/10.1111/j.1365-2672.1993.tb03019.x.

Gullberg, E., S. Cao, O.G. Berg, C. Ilbäck, L. Sandegren, and D. Hughes., and A.D. I. 2011. Selection of resistant bacteria at very low antibiotic concentrations. PLoS Pathog. 7:e1002158.

Hoyle, D. V., H. I. Knight, D. J. Shaw, K. Hillman, M. C. Pearce, J. C. Low, G. J. Gunn, and M. E. J. Woolhouse. 2004. Acquisition and epidemiology of antibiotic-resistant Escherichia coli in a cohort of newborn calves. J. Antimicrob. Chemother. 53:867-871. http:// dx.doi.org/10.1093/jac/dkh177.

Johnson-Ifearulundu, Y. J., and J. B. Kaneene. 1998. Managementrelated risk factors for $M$. paratuberculosis infection in Michigan, USA, dairy herds. Prev. Vet. Med. 37:41-54. http://dx.doi. org/10.1016/S0167-5877(98)00110-X.

Kaesbohrer, A., A. Schroeter, B.-A. Tenhagen, K. Alt, B. Guerra, and B. Appel. 2012. Emerging antimicrobial resistance in commensal Escherichia coli with public health relevance. Zoonoses Public Health 59:158-165. http://dx.doi.org/10.1111/j.18632378.2011.01451.x.

Khachatryan, A. R., D. D. Hancock, T. E. Besser, and D. R. Call. 2004. Role of calf-adapted Escherichia coli in maintenance of antimicrobial drug resistance in dairy calves. Appl. Environ. Microbiol. 70:752-757. http://dx.doi.org/10.1128/AEM.70.2.752757.2004 .

Klein-Jöbstl, D., E. Schornsteiner, E. Mann, M. Wagner, M. Drillich, and S. Schmitz-Esser. 2014. Pyrosequencing reveals diverse fecal microbiota in Simmental calves during early development. Front. Microbiol. 5: http://dx.doi.org/10.3389/fmicb.2014.00622.

Machuca, J., A. Briales, G. Labrador, P. Díaz-de-Alba, R. López-Rojas, F. Docobo-Pérez, L. Martínez-Martínez, J. Rodríguez-Baño, M. E. Pachón, A. Pascual, and J.-M. Rodríguez-Martínez. 2014. Interplay between plasmid-mediated and chromosomal-mediated fluoroquinolone resistance and bacterial fitness in Escherichio coli. J. Antimicrob. Chemother. 69:3203-3215. http://dx.doi. org $/ 10.1093 / \mathrm{jac} / \mathrm{dku} 308$.

Mackie, R. I., A. Sghir, and H. R. Gaskins. 1999. Developmental microbial ecology of the neonatal gastrointestinal tract. Am. J. Clin. Nutr. 69:1035S-1045S.

Marchese, A., E. Coppo, R. Barbieri, S. Zoppi, C. Pruzzo, F. Rossi, S. Bergagna, A. Dondo, and E. Debbia. 2012. Characterization of fluoroquinolone-resistant Escherichia coli causing septicemic colibacillosis in calves in Italy: Emergence of a multiresistant O78 clonal group. Microb. Drug Resist. 18:94-99. http://dx.doi. org/10.1089/mdr.2011.0053.

Mayer, M., A. Abenthum, J. M. Matthes, D. Kleeberger, M. J. Ege, C. Hölzel, J. Bauer, and K. Schwaiger. 2012. Development and genetic influence of the rectal bacterial microbiota of newborn calves. Vet. Microbiol. 161:179-185. http://dx.doi.org/10.1016/j. vetmic.2012.07.023.

Oikonomou, G., A. G. V. Teixeira, C. Foditsch, M. L. Bicalho, V. S. Machado, and R. C. Bicalho. 2013. Fecal microbial diversity in pre-weaned dairy calves as described by pyrosequencing of 
metagenomic 16S rDNA. Associations of Faecalibacterium species with health and growth. PLoS ONE 8:e63157. http://dx.doi. org/10.1371/journal.pone.0063157.

Pereira, R. V., J. D. Siler, J. C. Ng, M. A. Davis, Y. T. Grohn, and L. D. Warnick. 2014a. Effect of on-farm use of antimicrobial drugs on resistance in fecal Escherichia coli of preweaned dairy calves. J. Dairy Sci. 97:7644-7654. http://dx.doi.org/10.3168/jds.20148521.

Pereira, R. V. V., J. D. Siler, R. C. Bicalho, and L. D. Warnick. 2014b. In vivo selection of resistant E. coli after ingestion of milk with added drug residues. PLoS ONE 9:e115223. http://dx.doi. org/10.1371/journal.pone.0115223.

Pithua, P., L. A. Espejo, S. M. Godden, and S. J. Wells. 2013. Is an individual calving pen better than a group calving pen for preventing transmission of Mycobacterium avium subsp. paratuberculosis in calves? Results from a field trial. Res. Vet. Sci. 95:398-404. http://dx.doi.org/10.1016/j.rvsc.2013.03.014.

Rebelo, I. B. 2014. Risk of feeding calves with waste milk unfit for human consumption. MS Thesis. University of Lisboa, Lisboa, Portugal. [in Portugese]

Ruiz, J. 2003. Mechanisms of resistance to quinolones: Target alterations, decreased accumulation and DNA gyrase protection. J. Antimicrob. Chemother. 51:1109-1117. http://dx.doi.org/10.1093/ $\mathrm{jac} / \mathrm{dkg} 222$.

Sjögren, R. E. 1995. Thirteen-year survival study of an environmental Escherichia coli in field mini-plots. Water Air Soil Pollut. 81:315335. http://dx.doi.org/10.1007/BF01104018.

Swedres-Svarm. 2013. Use of antimicrobials and occurrence of antimicrobial resistance in Sweden. Public Health Agency of Sweden and National Veterinary Institute, Solna/Uppsala, Sweden.
Taylor, N. M., F. A. Clifton-Hadley, A. D. Wales, A. Ridley, and R. H. Davies. 2009. Farm-level risk factors for fluoroquinolone resistance in E. coli and thermophilic Campylobacter spp. on finisher pig farms. Epidemiol. Infect. 137:1121-1134. http://dx.doi. org $/ 10.1017 /$ S0950268808001854

Watson, E., S. Jeckel, L. Snow, R. Stubbs, C. Teale, H. Wearing, R. Horton, M. Toszeghy, O. Tearne, J. Ellis-Iversen, and N. Coldham. 2012. Epidemiology of extended spectrum beta-lactamase $E$. coli (CTX-M-15) on a commercial dairy farm. Vet. Microbiol. 154:339-346. http://dx.doi.org/10.1016/j.vetmic.2011.07.020.

Wiuff, C. J. Lykkesfeldt, F. M. Aarestrup, and O. Svendsen. 2002. Distribution of enrofloxacin in intestinal tissue and contents of healthy pigs after oral and intramuscular administrations. J. Vet. Pharmacol. Ther. 25:335-342. http://dx.doi.org/10.1046/j.13652885.2002.00430.x.

World Health Organization. 2012. Critically Important Antimicrobials for Human Medicine. 3rd rev. World Health Organization, Geneva, Switzerland

Wray, C., S. Furniss, and C. L. Benham. 1990. Feeding antibioticcontaminated waste milk to calves-effects on physical performance and antibiotic sensitivity of gut flora. Br. Vet. J. 146:80-87. http://dx.doi.org/10.1016/0007-1935(90)90080-M.

Wu, S., E. Chouliara, L. B. Jensen, and A. Dalsgaard. 2008. Evaluation of Petrifilm ${ }^{\text {TM }}$ Select E. coli Count Plate medium to discriminate antimicrobial resistant Escherichia coli. Acta Vet. Scand. 50:38 http://dx.doi.org/10.1186/1751-0147-50-38. 